\title{
Primary Lymphoma of Bone
}

National Cancer Institute

\section{Source}

National Cancer Institute. Primary Lymphoma of Bone. NCI Thesaurus. Code C6620.

A rare non-Hodgkin lymphoma or even more rarely, a Hodgkin lymphoma that arises from the bone, without lymph node or other extranodal involvement. The femur, spine, and pelvic bones are the most commonly affected sites. The majority of patients present with bone pain in the affected area. A single bone or multiple skeletal sites may be involved. The prognosis is related to the cell type and the stage of the disease. 